\title{
Standardization and Evaluation of an Anti-ZIKV IgM ELISA Assay for the Serological Diagnosis of Zika Virus Infection
}

\author{
Kanittha Sirikajornpan, ${ }_{1}$ Piyarat Suntarattiwong, ${ }^{2}$ Detchvijitr Suwanpakdee, ${ }^{3}$ Sutchana Tabprasit, ${ }^{4}$ Darunee Buddhari, ${ }^{1}$ \\ Butsaya Thaisomboonsuk, ${ }^{1}$ Chonticha Klungthong, ${ }^{1}$ Yongyuth Poolpanichupatam, ${ }^{1}$ Rome Buathong, ${ }^{5}$ Anon Srikiatkhachorn, 6,7 \\ Anthony Jones, ${ }^{1}$ Stefan Fernandez, ${ }^{1}$ and Taweewun Hunsawong ${ }^{1 *}$ \\ ${ }^{1}$ Department of Virology, Armed Forces Research Institute of Medical Sciences (AFRIMS), Bangkok, Thailand; ${ }^{2}$ Pediatrician, Infectious Diseases Unit, \\ Department of Pediatrics, Queen Sirikit National Institute of Child Health, Bangkok, Thailand; ${ }^{3}$ Department of Pediatrics, Phramongkutklao Hospital, \\ Bangkok, Thailand; ${ }^{4}$ Research Division, Royal Thai Army-Armed Forces Research Institute of Medical Sciences (RTA-AFRIMS), Bangkok, Thailand; \\ ${ }^{5}$ Department of Disease Control, Bureau of Epidemiology, Ministry of Public Health, Nonthaburi, Thailand; ${ }^{6}$ Institute for Immunology and Informatics, \\ University of Rhode Island, Providence, Rhode Island; ' Faculty of Medicine, King Mongkut's Institute of Technology Ladkrabang, Bangkok, Thailand
}

Abstract. Here, we describe the development of the in-house anti-Zika virus (ZIKV) IgM antibody capture ELISA (in-house ZIKV IgM ELISA) for the detection and diagnosis of acute ZIKV infections. We compared the in-house ZIKV IgM ELISA assay performance against two commercial kits, Euroimmun ZIKV IgM and InBios 2.0 ZIKV IgM ELISA. We tested the assays' ability to detect anti-ZIKV IgM using a well-defined serum sample panel. This panel included 80 ZIKV negative samples (20 negative, 20 found to be primary dengue virus [DENV][ infections, 20 secondary DENV infections, and 20 Japanese encephalitis virus [JEV] infections) and 67 ZIKV reverse transcriptase-polymerase chain reaction-positive acute serum samples. The OD values were calculated to enzyme immunoassay (EIA) unts by comparing them to weak positive controls. The results demonstrated the high sensitivity (88.06\%) and specificity (90.00\%) of our in-house ZIKV IgM ELISA and its $89.12 \%$ overall percentage agreement. The kappa values were deemed to be within excellent range and comparable to the InBios ZIKV IgM ELISA. Some cross-reactivity was observed among secondary DENV and JEV samples, and to a much lower extent, among primary DENV samples. These data indicate that our in-house ZIKV IgM ELISA is a reliable assay for the detection of anti-ZIKV IgM antibodies in serum.

\section{INTRODUCTION}

Zika virus (ZIKV) is a mosquito- borne pathogen, belonging to the Flaviviridae family, in the Spondweni sero-complex group. ${ }^{1}$ Zika virus was first isolated from a sentinel Rhesus monkey (Macaca mulatta) during a yellow fever study in the Zika forest near Entebbe, Uganda, in $1947 .^{2}$ In 1948, the virus was isolated from pooled Aedes africans circulating in the same forest. ${ }^{3}$ Zika virus has also been isolated from many other species of Aedes mosquitoes, ${ }^{4,5}$ including Aedes aegypti mosquitoes, ${ }^{6}$ the most significant vector of ZIKV transmission. Human-to-human transmission can also occur through blood transfusions, sexual contact, and vertically from mother to fetus. ${ }^{7}$

The first ZIKV infection in humans was reported in Nigeria in 1954. ${ }^{8}$ Zika virus infections usually manifest as asymptomatic or mild disease, most commonly accompanied by mild fever, arthralgia in small joints of the hands and feet, myalgia, headache, retro-orbital pain, conjunctivitis, and cutaneous maculopapular rash. Clinical diagnosis is often difficult because symptoms are shared by infections with other arboviruses, like dengue virus (DENV) and chikungunya virus (CHIKV). Abdominal pain, diarrhea, and arthritis can also appear in some cases of ZIKV infections. ${ }^{9}$ In 2007, a large outbreak of ZIKV disease took place in Yap state, Micronesia, infecting approximately $70 \%$ of the population. ${ }^{10}$ Since then, ZIKV has spread throughout various regions of the world, ${ }^{11-13}$ becoming a significant public health threat due to its association with significant neurological disorders in infants. ${ }^{14-16}$ Given the persistent circulation of ZIKV in some areas of the world, including Thailand, ${ }^{17}$ improvements in early detection

* Address correspondence to Taweewun Hunsawong, Department of Virology, USAMD-AFRIMS, 315/6 Ratchawithi Road, Ratchatewi, Bangkok 10400, Thailand. E-mail: taweewunh.fsn@afrims.org and responses, vector control programs, effective therapeutics and vaccines are needed to control infection and transmission. $^{18}$

The laboratory diagnosis of ZIKV mostly relies on the detection of viral RNA in whole blood (also serum and plasma), cerebrospinal fluid, saliva, urine, and semen. ${ }^{19,20}$ Zika virus viremia can be detected for up to 5 days after symptoms onset, peaking when clinical signs appear. ${ }^{10}$ Some evidence shows longer detectable periods of ZIKV viremia in urine and semen than in whole blood or saliva. ${ }^{21,22}$ Serological tests like ELISAs can provide a wider window for diagnosis because they are capable of detecting ZIKV antibodies' response early during an acute event and through convalescence. Zika virus IgM typically develops around 5 days after symptom onset and remains detectable for at least 12 weeks, whereas ZIKV IgG can be detected a few days later and remains detectable for at least 1 year. ${ }^{20}$ Despite its high cross-reactivity to other flaviviruses, ${ }^{23}$ detection of circulating ZIKV IgG antibodies continues to be widely used to identify prior ZIKV exposures in individuals. ${ }^{24}$ We seek to test the less cross-reactive ZIKV IgM antibodies as a tool to distinguish acute ZIKV infections from other flavivirus and extend the diagnostic window provided by reverse transcriptase-polymerase chain reaction (RT-PCR). Here, we standardized and characterized the performance of the in-house ZIKV IgM ELISA using patient sera and compared it with two commercial ELISA kits including Euroimmun ZIKV IgM ELISA (Euroimmun ELISA; Euroimmun AG, Lübeck, Germany) and InBios ZIKV detect 2.0 lgM capture ELISA (InBios MAC-ELISA; InBios international, Inc., Seattle, WA).

\section{MATERIALS AND METHODS}

Serum specimens. The human serum samples used in this study (Table 1) were obtained from Thai patients and confirmed by RT-PCR under a non-human subject research study 
TABLE 1

Sample panel characteristics of $N=147$ cases in pairs used in the study

\begin{tabular}{|c|c|c|c|c|c|c|c|}
\hline \multirow[b]{2}{*}{ Group } & \multirow[b]{2}{*}{ Label } & \multirow{2}{*}{$\begin{array}{c}\text { Cases } \\
(N=147)\end{array}$} & \multicolumn{3}{|c|}{ RT-PCR } & \multicolumn{2}{|c|}{ DENV/JEV MAC-ELISA } \\
\hline & & & DENV & JEV & ZIKV & DENV & JEV \\
\hline 1 & Negative & 20 & 0 & 0 & 0 & 0 & 0 \\
\hline 2 & $1^{\circ}$ DENV & 20 & 20 & 0 & 0 & 20 & 4 \\
\hline 3 & $2^{\circ}$ DENV & 20 & 20 & 0 & 0 & 20 & 0 \\
\hline 4 & JEV & 20 & 0 & 0 & 0 & 0 & 20 \\
\hline 5 & ZIKV & 67 & 0 & ND & 67 & 16 & 8 \\
\hline
\end{tabular}

approved by Walter Reed Army Institute of Research and local Institutional Review Boards. The deidentified samples consisted of a total of 147 pairs of acute and convalescent samples, including 67 ZIKV RT-PCR-positive samples and 80 ZIKV RT-PCR-negative samples. The RT-PCR-negative samples were composed of samples with no evidence of flavivirus infection $(N=20)$, primary DENV infections $(N=20)$, or secondary DENV infections $(N=20)$ and were Japanese encephalitis virus (JEV) IgM positive $(N=20)$.

Viral RNA detection by RT-PCR. Viral RNA was extracted using QIAamp viral RNA mini kit (Qiagen, Hilden, Germany) according to the manufacturer's instruction. Samples were tested by nested RT-PCR for detection of DENV and JEV ${ }^{25}$ and real-time RT-PCR for detection of ZIKV. For ZIKV realtime RT-PCR, the method was modified from Lanciotti et al. ${ }^{20}$ by using the two primer/probe sets. The first set includes ZIKV 1086 forward primers, ZIKV 1162c reverse primers, and a ZIKV 1107-FAM probe, ${ }^{26}$ and the second set includes ZIKV 4434 forward primers, ZIKV 4524c reverse primers, and a ZIKV $4479 c-F A M$ probe. ${ }^{27}$ All real-time assays were performed by using the SuperScript III Platinum One-Step Quantitative RT-PCR System (Invitrogen, Waltham, MA) with amplification using the Applied Biosystems 7500 Fast Real-Time PCR systems (Life Technologies, Carlsbad, CA) following the manufacturer's protocol.

Anti-DENV/JEV IgM and IgG ELISA. Anti-DENV/JEV IgM and IgG ELISAs were performed in three independent experiments following procedure described elsewhere. ${ }^{28,29}$ Briefly, flat-bottom microplates were coated with $100 \mu \mathrm{L} /$ well of $1: 1,600$ dilution of goat anti-human IgM or IgG (KPL, Gaithersburg, MD) in 0.018 M carbonate buffer ( $\mathrm{pH} 9.0)$. After overnight incubation at $4{ }^{\circ} \mathrm{C}$, the plates were washed with phosphate buffered saline (PBS) ( $\mathrm{pH} \mathrm{7.4)} \mathrm{containing} \mathrm{0.5 \%} \mathrm{Tween} 20$ (PBS-T). Next, $50 \mu \mathrm{L} /$ well of 1:100 dilution of test serum, negative control (NC), weak positive control (WPC), and strong positive control (SPC) in PBS were added and incubated overnight at $4^{\circ} \mathrm{C}$. After washing with PBS-T, $50 \mu \mathrm{L} /$ well of sucrose acetone extracted suckling mouse brain DENV (pooled DENV antigen: DENV-1 [Hawaii], DENV [NGC], DENV-3 [H87], and DENV-4 [H241]) and JEV (JaGAr01) antigens were added into DENV (IgM/lgG) and JEV (IgM/lgG) plates, respectively. After incubation for $2 \mathrm{~h}$ at room temperature, $30 \mu \mathrm{L} /$ well of human anti-flavivirus IgG-horseradish peroxidase conjugated was added and incubated for 1 hour at $37^{\circ} \mathrm{C}$. After washing with PBS-T, $100 \mu \mathrm{L} /$ well of TMB substrate (KPL, Gaithersburg, MD) was added and incubated for 10-30 min. The reaction was stopped by adding $50 \mu \mathrm{L} /$ well of $0.2 \mathrm{M}$ sulfuric acid.
The absorbance (optical density [OD]) was measured at a wavelength of $450 \mathrm{~nm}$ (SoftMax Pro Software, Molecular Devices, San Jose, CA). A valid assay should provide OD values at $<0.100,0.400-0.600$, and $>0.600$ for NC, WPC, and SPC, respectively. EIA units of tested serum are equal to $100 \times$ $\left[\left(O D_{\text {Test }}-O D_{N C}\right) /\left(O D_{W P C}-O D_{N C}\right)\right]$; EIA units of $\operatorname{lgM} \geq 40$ were used as a positive cut-off value. Evidence of dengue infection was classified by a ratio of DENV IgM/JEV IgM $\geq$ 1.0 , and JEV infection when the ratio was $<1.0$. Primary DENV infection was interpreted when the ratio of DENV IgM/ DENV IgG was $\geq 1.8$, and secondary DENV infection was considered when the ratio was $<1.8$.

In-house ZIKV IgM ELISA. A capture ELISA method was used to develop an in-house ZIKV IgM ELISA. Briefly, flatbottom microplates were coated with $100 \mu \mathrm{L} /$ well of $1: 1,600$ dilution of goat anti-human IgM (KPL, Gaithersburg, MD) in $0.018 \mathrm{M}$ carbonate buffer ( $\mathrm{pH}$ 9.0). After overnight incubation at $4{ }^{\circ} \mathrm{C}$, the plates were washed with PBS $(\mathrm{pH} 7.4)$ containing $0.5 \%$ Tween 20 (PBS-T). Next, $50 \mu \mathrm{L} /$ well of 1:100 dilution of test serum, NC, WPC and SPC in PBS were added and incubated overnight at $4^{\circ} \mathrm{C}$. After washing with PBS-T, $50 \mu \mathrm{L} /$ well of sucrose acetone extracted suckling mouse brain ZIKV (MR766; Uganda, 1947) antigen (50-100 HA units) was added to the microplate. After incubation for $2 \mathrm{~h}$ at $37^{\circ} \mathrm{C}, 30 \mu \mathrm{L} /$ well of human anti-flavivirus IgG-HRP conjugated was added and incubated for $1 \mathrm{~h}$ at $37^{\circ} \mathrm{C}$. After washing with PBS-T, 100 $\mu \mathrm{L} /$ well of TMB substrate (KPL) was added and incubated for 10-30 min. The reaction was stopped by adding $50 \mu \mathrm{L} /$ well of $0.2 \mathrm{M}$ sulfuric acid. The absorbance at OD450 was measured and calculated for EIA units as previously described. ${ }^{28,29}$ The WPC for anti-ZIKA IgM ELISA was obtained from the serum of a rhesus monkey (Macaca mulatta) 15 days after inoculation with $5 \times 10^{6}$ PFU of ZIKV (MR766; Uganda, 1947).

Euroimmun anti-Zika virus IgM ELISA (Euroimmun ZIKV-IgM). The Euroimmun ZIKA IgM ELISA test kit was designed to detect specific IgM against recombinant ZIKV NS1 coated on the plate. The Euroimmun Zika Virus ELISA kits (Cat. No. El2668-9601M) were produced by Euroimmun AG (Lübeck, Germany). The assays were carried out according to the manufacturer's instructions. ${ }^{30}$

InBios ZIKV detect $^{\mathrm{TM}} 2.0$ IgM capture ELISA (InBios ZIKV-IgM). The InBios ZIKV IgM ELISA detects IgM antibodies targeting the recombinant ZIKV envelope glycoproteins (Cat. No. ZKM2-1). The InBios ZIKV-IgM ELISA kit was produced by the InBios international, Inc. (Seattle, WA) and performed according to the manufacturer's instructions. ${ }^{31}$

Data analysis. Prism-GraphPad (GraphPad software Inc., La Jolla, CA) was used to create the receiver operating characteristic (ROC) curve to identify an appropriate cut-off value for anti-ZIKV IgM ELISA. The assay performance comparison to commercial kits (assay agreement) was evaluated by Kappa values, indicating excellent agreement if $>0.75$, fair agreement if $0.40-0.75$, and poor agreement if $<0.40$. $^{32}$

\section{RESULTS}

Receiver operating characteristic curve of anti-ZIKV IgM ELISA. Table 1 shows the composition and characteristics of the serum panel used. All of the samples were tested by RT-PCR and DENV/JEV IgM/lgG ELISA. The ROC curve (Figure 1) was used to determine an optimal EIA 


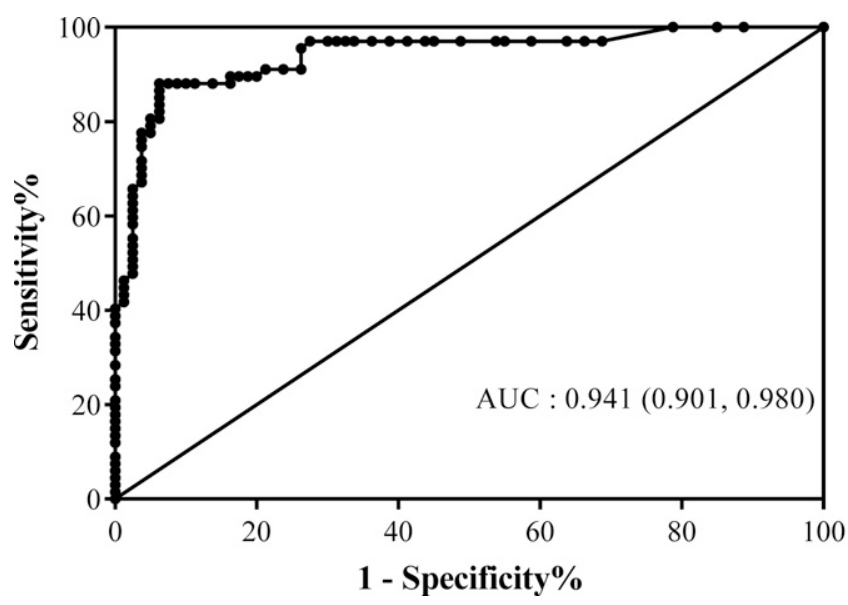

FIGURE 1. Receiver operating characteristic (ROC) curve of the In-house ZIKV IgM ELISA. The graph shows the area under the ROC (AUC) value and the $95 \%$ confidence intervals in parentheses. The sensitivity and specificity values correspond to the points in the plots. The ROC curve was constructed using 80 ZIKV RT-PCR negative and 67 cases of ZIKV RT-PCR positive samples.

unit cut-off point to distinguish between negative and positive samples and still provide diagnosis accuracy. ${ }^{33,34}$ In some instances, ZIKV RT-PCR-positive samples were found to be cross-reactive to DENV $(N=16)$ or JEV $(N=8)$ when tested with the DEN/JEV IgM/lgG ELISA. The area under the ROC curve (AUC) value of the in-house ZIKV IgM ELISA was 0.941 , reflecting excellent diagnostic accuracy. A ROC curve pointing to a cut-off line at $40 \mathrm{EIA}$ units is considered optimal, providing assay sensitivity and specificity of up to $88.06 \%$ and $90.00 \%$, respectively. The EIA unit distribution of validated samples is shown in a scatter plot with cut-off line (Figure 2). Most of ZIKV RT-PCR-positive sera samples showed higher levels of ZIKV IgM antibody binding than other groups. Some cross-reactivity among flavivirus was observed because some of DENV (mostly secondary) and JEV samples appear over the cut-off line.

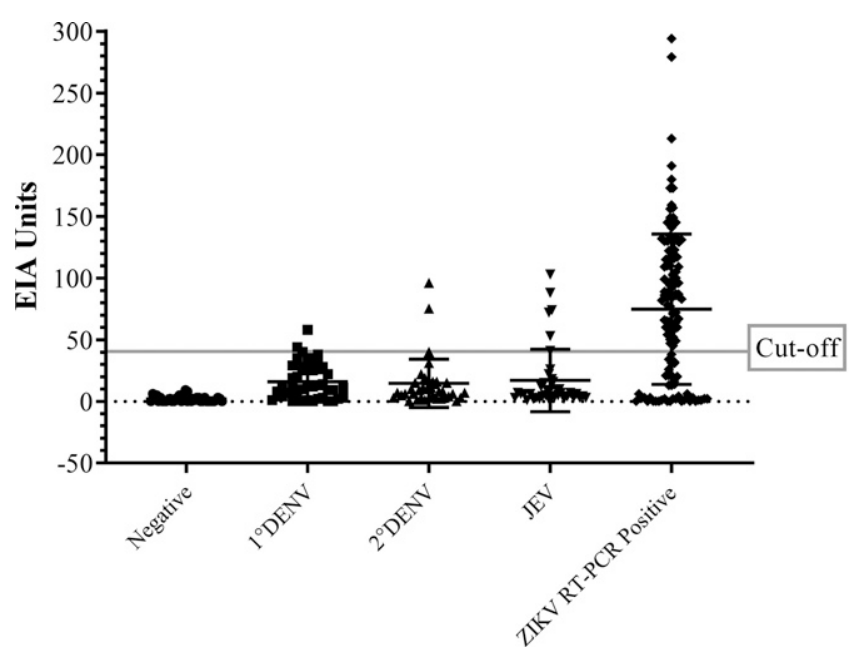

FIGURE 2. The EIA unit distribution of validated samples by using the In-house ZIKV IgM ELISA. The cut-off was set at 40 EIA unit.
The detection of ZIKV IgM by anti-ZIKV IgM ELISAs. The performance of the in-house ZIKV IgM ELISA was compared with two commercial ELISA kits: Euroimmun ZIKV IgM ELISA and InBios ZIKV IgM ELISA. Table 2 shows the serum panel results done by all three ZIKV IgM ELISA tests. The negative ZIKV samples were found to be $90 \%(72 / 80)$ negative when tested by the in-house ZIKV IgM ELISA. Fifty-nine of 67 (88.06\%) ZIKV RT-PCR-positive samples were found to be positive when tested by the in-house ZIKV IgM ELISA. The Euroimmun ZIKV IgM ELISA showed 98.75\% (79/80) agreement with the ZIKV RT-PCR-negative samples but only $13.43 \%$ (9/67) agreement with the ZIKV RT-PCR-positive samples, with an additional 4.48\% (3/67) labeled as borderline. The InBios ZIKV IgM ELISA had a $94.03 \%$ (63/67) agreement with the ZIKV RT-PCR-positive samples with an additional one sample (1.5\%) identified as "other flavivirus." The InBios ZIKV IgM ELISA also had a 91.25\% (73/80) agreement ZIKV RT-PCR-negative samples, which also includes the correct assessment of $47 / 60$ (78.33\%) as other flaviviruses.

Overall sensitivity and specificity. Table 3 shows the case distribution and comparison of sensitivity, specificity, overall agreement, and Kappa assessment values $(95 \% \mathrm{Cl})$ of the in-house ZIKV IgM ELISA assay to two commercial ELISA kits (Euroimmun ZIKV IgM ELISA and InBios ZIKV IgM ELISA). By using ZIKV RT-PCR and DENV/JE IgM ELISA as reference standard methods, the sensitivities of the in-house ZIKV-IgM ELISA, Euroimmun ZIKV IgM ELISA, and InBios ZIKV IgM ELISA were $88.06 \%$ (59/67), $10.45 \%$ (7/67), and $94.03 \%$ (63/67), respectively. The assay specificities were $90.00 \%$ (72/80), 98.75\% (79/80), and 83.75\% (67/80) for in-house ZIKV-IgM ELISA, Euroimmun ZIKV IgM ELISA, and InBios ZIKV IgM ELISA, respectively. The percentage overall agreement to the ZIKV RT-PCR and DENV/JE EIA of the in-house ZIKV IgM ELISA, Euroimmun ZIKV IgM ELISA, and InBios ZIKV IgM ELISA were 89.12\% (131/147), 58.5\% (86/147), and $88.4 \%$ (130/147), respectively. Kappa assessment values define the in-house ZIKV-IgM ELISA (Kappa value: 0.83) and InBios ZIKV IgM ELISA (Kappa value: 0.81) as excellent, whereas the Euroimmun ZIKV IgM (Kappa value: 0.45) was classified as fair.

\section{DISCUSSION}

Serological diagnosis of ZIKV infection is challenging, often leading to misinterpretation ${ }^{35}$ due to the cross-reactive nature of antibodies elicited by infection to other flaviviruses bearing common antigenic determinants or by vaccination. ${ }^{20,36}$ Reverse transcriptase-polymerase chain reaction is the most reliable assay today for ZIKV detection and diagnosis but is limited by the short-lived presence of viral RNA in acute serum, often lasting only 3-5 days after symptoms. ${ }^{19,20}$ Validated serological assays of high specificity and sensitivity, capable of detection of ZIKV-specific antibodies circulating during the acute and early convalescence phases, would expand the window of detection in support of ZIKV diagnosis and treatment.

In this study, we developed a specific in-house ZIKV IgM ELISA and measured its specificity and sensitivity using a well-defined serum panel consisting of ZIKV RT-PCR-positive serum samples and ZIKV RT-PCR-negative serum samples of other or unknown etiologies. Receiver operating 
TABLE 2

In-house ZIKV IgM, Euroimmun ZIKV IgM, and InBios ZIKV IgM ELISAs test results

\begin{tabular}{|c|c|c|c|c|c|c|c|}
\hline \multirow[b]{2}{*}{ ELISAs } & \multirow[b]{2}{*}{ Results } & \multirow[b]{2}{*}{$\mathrm{ZIKV}(N=67)$} & \multicolumn{3}{|c|}{ Others flavivirus } & \multirow{2}{*}{$\begin{array}{l}\text { Negative } \\
(N=20)\end{array}$} & \multirow{2}{*}{$\begin{array}{c}\text { Total } \\
(N=147\end{array}$} \\
\hline & & & $1^{\circ}$ DENV $(N=20)$ & $2^{\circ}$ DENV $(N=20)$ & JEV $(N=20)$ & & \\
\hline \multirow[t]{2}{*}{ In-house ZIKV IgM } & ZIKV & 59 & 2 & 2 & 4 & 0 & 147 \\
\hline & Negative & 8 & 18 & 18 & 16 & 20 & \\
\hline \multirow[t]{3}{*}{ Euroimmun ZIKV IgM } & ZIKV & 9 & 0 & 0 & 1 & 0 & 147 \\
\hline & Borderline & 3 & 0 & 0 & 0 & 0 & \\
\hline & Negative & 55 & 20 & 20 & 19 & 20 & \\
\hline \multirow[t]{3}{*}{ InBios 2.0 ZIKV IgM } & ZIKV & 63 & 0 & 1 & 6 & 0 & 147 \\
\hline & Other flavivirus & 1 & 20 & 18 & 9 & 0 & \\
\hline & Negative & 3 & 0 & 1 & 5 & 20 & \\
\hline
\end{tabular}

IgM = immunoglobulin M; ZIKV = Zika virus.

characteristic curve analysis using the differences between true positive rate (sensitivity) and false positive rate ( 1 = specificity) showed that 40 EIA units represents the optimal cut-off point. ${ }^{33}$ Using this cut-off point, the AUC approximates 1 (AUC =0.941) and indicates excellent diagnostic capabilities and accuracy of our in-house ZIKV IgM ELISA, with $88.06 \%$ sensitivity and $90.00 \%$ specificity.

We used the in-house DENV/JEV IgM/IgG ELISA ${ }^{28,29}$ as a model to develop the in-house ZIKV IgM ELISA. Evidence of ZIKV infection was classified when the ratio of ZIKV IgM/ DENV IgM and ZIKV IgM/JEV IgM was $\geq 1.0$. Using this criteria, we found ease in distinguishing between negative/primary DENV and ZIKV samples. However, we found low levels of cross-reactivity to ZIKV IgM when testing secondary DENV samples $(1.49 \%, 1 / 67)$ or JEV $(5.97 \%, 4 / 67)$.

The finding of negative in-house ZIKV IgM ELISA results among ZIKV RT-PCR-positive samples (8/67) could be due to improper sample collection times for IgM detection, including early collection of acute and/or late collection of the convalescent samples. Even though ZIKV cross-reactivity has been observed in the JEV samples, anti-JEV IgM read-outs were higher than anti-ZIKV IgM antibodies, facilitating assay interpretation. The JEV immunization program has been implemented in Thailand since $1990,{ }^{37}$ and it has been reported that the overall protective immunity to JEV was up to $75 \%$ in Thai people. ${ }^{38}$ We were unable to search the medical or vaccination history of our sample donors. However, it is a reasonable expectation that JEV immunization would be a possible cause of the high anti-JEV IgM levels, which are cross-reactive and interfering with other flavivirus serological tests. ${ }^{39}$ Ultimately, there is a risk of false positives when ZIKV IgM ELISAs are used in areas where ZIKV co-circulates with various flaviviruses. Nonetheless, our data show that the in-house ZIKV IgM ELISA is highly specific, even when testing samples from patients with primary flavivirus infections.

Evaluations of various Zika ELISA assays have been published elsewhere. ${ }^{40,41}$ In general, envelop-based Zika IgM ELISA assays increase detection rates significantly over NS-1-based assays and provide a larger diagnostic window. ${ }^{42}$ These observations are reflected in the performance described here for the Euroimmun ZIKV IgM ELISA, a NS1-based assay with moderate results. In contrast, ZIKV IgM ELISA (using suckling mouse brain extracted crude antigen) and the InBios ZIKV IgM ELISA (recombinant envelope protein) performed substantially better. Euroimmun has recently recommended the use of the combined ZIKV IgA/lgM to improve its sensitivity. ${ }^{43}$

In conclusion, our study demonstrates that the in-house ZIKV IgM ELISA can be an important tool for detecting ZIKV infections in humans. The assay is an affordable and reliable option for diagnosis of ZIKV patients, especially in flavivirusendemic countries. A limitation in the use of the in-house ZIKV-IgM ELISA is that its interpretation requires the use of

TABLE 3

Comparison results of In-house ZIKV IgM ELISA, Euroimmun ZIKV IgM, and InBios ZIKV IgM ELISAs

\begin{tabular}{|c|c|c|c|c|c|c|c|c|c|}
\hline \multirow[b]{2}{*}{ ELISA } & \multirow[b]{2}{*}{ Result $^{\star}$} & \multicolumn{3}{|c|}{ Validation samples } & \multirow[b]{2}{*}{ Total } & \multirow[b]{2}{*}{$\begin{array}{l}\text { \% Sensitivity } \\
(95 \% \mathrm{Cl})\end{array}$} & \multirow[b]{2}{*}{$\begin{array}{l}\text { \% Specificity } \\
(95 \% \mathrm{Cl})\end{array}$} & \multirow[b]{2}{*}{$\begin{array}{c}\% \text { Overall } \\
\text { agreement }(95 \% \mathrm{Cl})\end{array}$} & \multirow[b]{2}{*}{$\begin{array}{c}\text { Kappa } \\
\text { assessment (95\% Cl) }\end{array}$} \\
\hline & & ZIKV & Other & NEG & & & & & \\
\hline \multirow[t]{4}{*}{ In-house ZIKV-IgM } & ZIKV & 59 & 8 & 0 & 67 & $59 / 67$ & $72 / 80$ & $131 / 147$ & \\
\hline & Other (a) & 0 & 52 & 0 & 52 & $88.06 \%$ & $90.00 \%$ & $89.12 \%$ & 0.83 \\
\hline & Negative & 8 & 0 & 20 & 28 & $(77.9-94.1)$ & $(81.3-95.1)$ & (83.0-93.3) & $\begin{array}{l}\text { (0.74-0.91) } \\
\text { excellence }\end{array}$ \\
\hline & Total & 67 & 60 & 20 & 147 & & & & \\
\hline \multirow[t]{4}{*}{ Euroimmun ZIKV IgM } & ZIKV & 9 & 1 & 0 & 10 & $9 / 67$ & $79 / 80$ & $88 / 147$ & \\
\hline & Other (b) & 3 & 59 & 0 & 62 & $13.43 \%$ & $98.75 \%$ & $59.86 \%$ & 0.45 \\
\hline & Negative & 55 & 0 & 20 & 75 & $(7.0-23.8)$ & $(92.6-100)$ & $(51.8-67.4)$ & $(0.34-0.56)$ fair \\
\hline & Total & 67 & 60 & 20 & 147 & & & & \\
\hline \multirow[t]{4}{*}{ InBios 2.0 ZIKV IgM } & ZIKV & 63 & 7 & 0 & 70 & $63 / 67$ & $73 / 80$ & $130 / 147$ & \\
\hline & Other (c) & 1 & 47 & 0 & 48 & $94.03 \%$ & $91.25 \%$ & $88.44 \%$ & 0.81 \\
\hline & Negative & 3 & 6 & 20 & 29 & $(85.2-98.1)$ & $(82.8-96.0)$ & $(82.2-92.7)$ & $(0.73-0.90)$ excellence \\
\hline & Total & 67 & 60 & 20 & 147 & & & & \\
\hline
\end{tabular}


DENV and JEV antibodies ELISAs, which may limit its usability in some laboratories.

Received February 9, 2021. Accepted for publication May 24, 2021.

Published online August 2, 2021.

Financial support: The research was funded by a grant from the Armed Forces Health Surveillance Branch (AFHSB) and its Global Emerging Infectious Surveillance (GEIS) Section, United States, under grant number P0108_19_AF_07 for fiscal year 2019.

Disclaimer: This material has been reviewed by the Walter Reed Army Institute of Research. There is no objection to its presentation and/or publication. The opinions or assertions contained herein are the private views of the authors and are not to be construed as official or as reflecting true views of the Department of the Army or the Department of Defense.

Authors' addresses: Kanittha Sirikajornpan, Butsaya Thaisomboonsuk, Chonticha Klungthong, Yongyuth Poolpanichupatam, Anthony Jones, Stefan Fernandez, and Taweewun Hunsawong, Department of Virology, Armed Forces Research Institute of Medical Sciences (AFRIMS), Bangkok, Thailand, E-mails: kanitthas.ca@afrims.org, daruneet.fsn@afrims.org, butsayat.ca@afrims.org, chontichak.fsn@ afrims.org, yongyuthp.fsn@afrims.org, anthony.jones.mil@afrims.org, stefan.fernandez.mil@afrims.org, and taweewunh.fsn@afrims.org. Piyarat Suntarattiwong, Pediatrician, Infectious Diseases Unit, Department of Pediatrics, Queen Sirikit National Institute of Child Health, Bangkok, Thailand, E-mail: drjunesunta@yahoo.com. Detchvijitr Suwanpakdee, Department of Pediatrics, Phramongkutklao Hospital, Bangkok, Thailand, E-mail: detchvijitr@ gmail.com. Sutchana Tabprasit, Research Division, Royal Thai Army, Armed Forces Research Institute of Medical Sciences (RTAAFRIMS), Bangkok, Thailand, E-mail: sutchanat.rta@afrims.org. Rome Buathong, Department of Disease Control, Bureau of Epidemiology, Ministry of Public Health, Nonthaburi, Thailand, E-mail: romebua@hotmail.com. Anon Srikiatkhachorn, Institute for Immunology and Informatics, University of Rhode Island, Providence, RI, and Faculty of Medicine, King Mongkut's Institute of Technology Ladkrabang, Bangkok, Thailand, E-mail: anons.gst@ afrims.org.

This is an open-access article distributed under the terms of the Creative Commons Attribution (CC-BY) License, which permits unrestricted use, distribution, and reproduction in any medium, provided the original author and source are credited.

\section{REFERENCES}

1. Kuno G, Chang GJ, Tsuchiya KR, Karabatsos N, Cropp CB, 1998. Phylogeny of the genus Flavivirus. J Virol 72: 73-83.

2. Dick GW, Kitchen SF, Haddow AJ, 1952. Zika virus. I. Isolations and serological specificity. Trans $R$ Soc Trop Med Hyg 46: 509-520.

3. Dick GW, 1952b. Zika virus. II. Pathogenicity and physical properties. Trans R Soc Trop Med Hyg 46: 521-534.

4. Haddow AD, Schuh AJ, Yasuda CY, Kasper MR, Heang V, Huy R, Guzman H, Tesh RB, Weaver SC, 2012. Genetic characterization of Zika virus strains: geographic expansion of the Asian lineage. PLoS Negl Trop Dis 6: e1477.

5. McCrae AW, Kirya BG, 1982. Yellow fever and Zika virus epizootics and enzootics in Uganda. Trans $R$ Soc Trop Med Hyg 76: $552-562$

6. Marchette NJ, Garcia R, Rudnick A, 1969. Isolation of Zika virus from Aedes aegypti mosquitoes in Malaysia. Am J Trop Med Hyg 18: 411-415.

7. Slavov SN, Otaguiri KK, Kashima S, Covas DT, 2016. Overview of Zika virus (ZIKV) infection in regards to the Brazilian epidemic. Braz J Med Biol Res 49: e5420.

8. Macnamara FN, 1954. Zika virus: a report on three cases of human infection during an epidemic of jaundice in Nigeria. Trans R Soc Trop Med Hyg 48: 139-145.

9. Zammarchi $L$ et al., 2015. Zika virus infections imported to Italy: clinical, immunological and virological findings, and public health implications. J Clin Virol 63: 32-35.
10. Duffy MR et al., 2009. Zika virus outbreak on Yap Island, Federated States of Micronesia. N Engl J Med 360: 2536-2543.

11. Cao-Lormeau VM, Roche C, Teissier A, Robin E, Berry AL, Mallet HP, Sall AA, Musso D, 2014. Zika virus, French Polynesia, South Pacific, 2013. Emerg Infect Dis 20: 1085-1086.

12. Heang V, Yasuda CY, Sovann L, Haddow AD, Travassos da Rosa AP, Tesh RB, Kasper MR, 2012. Zika virus infection, Cambodia, 2010. Emerg Infect Dis 18: 349-351.

13. Fonseca K, Meatherall B, Zarra D, Drebot M, MacDonald J, Pabbaraju K, Wong S, Webster P, Lindsay R, Tellier R, 2014. First case of Zika virus infection in a returning Canadian traveler. Am J Trop Med Hyg 91: 1035-1038.

14. Cugola FR et al., 2016. The Brazilian Zika virus strain causes birth defects in experimental models. Nature 534: 267-271.

15. Ventura CV, Maia M, Bravo-Filho V, Gois AL, Belfort R Jr, 2016. Zika virus in Brazil and macular atrophy in a child with microcephaly. Lancet 387: 228.

16. WHO, 2016. Neurological Syndrome and Congenital Anomalies. Zika Situation Report. Geneva, Switzerland: World Health Organization. Available at: http://apps.who.int/iris/bitstream/10665/ 204348/1/zikasitrep_5Feb2016_eng.pdf?ua=1. Accessed February $5,2016$.

17. Ruchusatsawat K, Wongjaroen $\mathrm{P}$, Posanacharoen A, RodriguezBarraquer I, Sangkitporn S, Cummings DAT, Salje H, 2019. Long-term circulation of Zika virus in Thailand: an observational study. Lancet Infect Dis 19: 439-446.

18. WHO, 2019. ZIKA Epidermiology Update. Geneva, Switzerland: World Health Organization. Available at: https://www.who.int/ emergencies/diseases/zika/zika-epidemiology-update-july2019.pdf?ua $=1$. Accessed July 2019.

19. Berthet N, Nakoune E, Kamgang B, Selekon B, Descorps-Declere S, Gessain A, Manuguerra JC, Kazanji M, 2014. Molecular characterization of three Zika flaviviruses obtained from sylvatic mosquitoes in the Central African Republic. Vector Borne Zoonotic Dis 14: 862-865.

20. Lanciotti RS, Kosoy OL, Laven JJ, Velez JO, Lambert AJ, Johnson AJ, Stanfield SM, Duffy MR, 2008. Genetic and serologic properties of Zika virus associated with an epidemic, Yap State, Micronesia, 2007. Emerg Infect Dis 14: 1232-1239.

21. Gourinat AC, O'Connor O, Calvez E, Goarant C, Dupont-Rouzeyrol M, 2015. Detection of Zika virus in urine. Emerg Infect Dis 21: 84-86.

22. Medina FA et al., 2019. Duration of the presence of infectious Zika virus in semen and serum. $J$ Infect Dis 219: 31-40.

23. Medialdea-Carrera R, Levy F, Castanha P, Sequeira PCd, Brasil $P$, Lewis-Ximenez LL, Turtle L, Solomon T, Filippis AMBd, Brown DW, Griffiths MJ, 2020. A systematic evaluation of $\operatorname{IgM}$ and IgG antibody assay accuracy in diagnosing acute Zika virus infection in Brazil: lessons relevant to emerging infections. BioRxiv.

24. Sumita LM, Rodrigues JP, Ferreira NE, Felix AC, Souza NC, Machado CM, Junior HF, 2016. Detection of human anti-Zika virus IgG by ELISA using an antigen from in vitro infected vero cells: preliminary results. Rev Inst Med Trop São Paulo 58: 89.

25. Jarman RG, Nisalak A, Anderson KB, Klungthong C, Thaisomboonsuk B, Kaneechit W, Kalayanarooj S, Gibbons RV, 2011. Factors influencing dengue virus isolation by $\mathrm{C} 6 / 36$ cell culture and mosquito inoculation of nested PCR-positive clinical samples. Am J Trop Med Hyg 84: 218-223.

26. Colombo TE et al., 2018. Zika detection: comparison of methodologies. Braz J Microbiol 49: 144-147.

27. Waggoner JJ, Pinsky BA, 2016. Zika virus: diagnostics for an emerging pandemic threat. J Clin Microbiol 54: 860-867.

28. Innis BL, Nisalak A, Nimmannitya S, Kusalerdchariya S, Chongswasdi V, Suntayakorn S, Puttisri P, Hoke CH, 1989. An enzyme-linked immunosorbent assay to characterize dengue infections where dengue and Japanese encephalitis co-circulate. Am J Trop Med Hyg 40: 418-427.

29. Burke DS, Nisalak A, Gentry MK, 1987. Detection of flavivirus antibodies in human serum by epitope-blocking immunoassay. $J$ Med Virol 23: 165-173.

30. Matheus S, Talla C, Labeau B, de Laval F, Briolant S, Berthelot L, Vray M, Rousset D, 2019. Performance of 2 commercial 
serologic tests for diagnosing Zika virus infection. Emerg Infect Dis 25: 1153-1160.

31. Basile AJ, Ao J, Horiuchi K, Semenova V, Steward-Clark E, Schiffer J, 2019. Performance of InBios ZIKV Detect 2.0 IgM Capture ELISA in two reference laboratories compared to the original ZIKV Detect IgM Capture ELISA. J Virol Methods 271: 113671.

32. Fleiss JL, Levin B, Paik MC, 2004. Statistical Methods for Rates and Proportions. Hoboken, NJ: Wiley.

33. Parikh R, Mathai A, Parikh S, Chandra Sekhar G, Thomas R, 2008. Understanding and using sensitivity, specificity and predictive values. Indian J Ophthalmol 56: 45-50.

34. Raez-Bravo A et al., 2016. Evaluation of three enzyme-linked immunosorbent assays for sarcoptic mange diagnosis and assessment in the Iberian ibex, Capra pyrenaica. Parasit Vectors 9: 558 .

35. Steinhagen $\mathrm{K}$ et al., 2016. Serodiagnosis of Zika virus (ZIKV) infections by a novel NS1-based ELISA devoid of cross-reactivity with dengue virus antibodies: a multicohort study of assay performance, 2015 to 2016. Euro Surveill 1: 30426.

36. Gyurech D, Schilling J, Schmidt-Chanasit J, Cassinotti P, Kaeppeli F, Dobec M, 2016. False positive dengue NS1 antigen test in a traveller with an acute Zika virus infection imported into Switzerland. Swiss Med Wkly 146: w14296.
37. Chunsuttiwat $S$, Warachit $P, 1995$. Japanese encephalitis in Thailand. Southeast Asian J Trop Med Public Health 26 (Suppl 3): 43-46.

38. Yoocharoan P, A-neugoolpipat A, Anantapreecha S, Tharmaphornpilas $P, 2009$. Seroprevalence of immunity against Japanese encepalitis in Thai population. Dis Control J 35: 276-284.

39. Saito Y, Moi ML, Takeshita N, Lim CK, Shiba H, Hosono K, Saijo M, Kurane I, Takasaki T, 2016. Japanese encephalitis vaccinefacilitated dengue virus infection-enhancement antibody in adults. BMC Infect Dis 16: 578.

40. Safronetz D et al., 2017. Evaluation of 5 commercially available Zika virus immunoassays. Emerg Infect Dis 23: 1577-1580.

41. Granger $D$ et al., 2017. Serologic testing for Zika virus: comparison of three Zika virus IgM-screening enzyme-linked immunosorbent assays and initial laboratory experiences. J Clin Microbiol 55: 2127-2136.

42. Bozza FA et al., 2019. Differential shedding and antibody kinetics of Zika and Chikungunya viruses, Brazil. Emerg Infect Dis 25: 311-315.

43. Warnecke JM, Lattwein E, Saschenbrecker S, Stocker W, Schlumberger W, Steinhagen K, 2019. Added value of IgA antibodies against Zika virus non-structural protein 1 in the diagnosis of acute Zika virus infections. J Virol Methods 267: 8-15. 